\title{
A Numerical Method for Partial Differential Algebraic Equations Based on Differential Transform Method
}

\author{
Murat Osmanoglu and Mustafa Bayram \\ Department of Mathematical Engineering, Chemical and Metallurgical Faculty, Yildiz Technical University, \\ Esenler 34210, Istanbul, Turkey
}

Correspondence should be addressed to Mustafa Bayram; msbayram@yildiz.edu.tr

Received 14 January 2013; Accepted 28 February 2013

Academic Editor: Adem Kiliçman

Copyright (C) 2013 M. Osmanoglu and M. Bayram. This is an open access article distributed under the Creative Commons Attribution License, which permits unrestricted use, distribution, and reproduction in any medium, provided the original work is properly cited.

We have considered linear partial differential algebraic equations (LPDAEs) of the form $A u_{t}(t, x)+B u_{x x}(t, x)+C u(t, x)=f(t, x)$, which has at least one singular matrix of $A, B \in \mathbb{R}^{n \times n}$. We have first introduced a uniform differential time index and a differential space index. The initial conditions and boundary conditions of the given system cannot be prescribed for all components of the solution vector $u$ here. To overcome this, we introduced these indexes. Furthermore, differential transform method has been given to solve LPDAEs. We have applied this method to a test problem, and numerical solution of the problem has been compared with analytical solution.

\section{Introduction}

The partial differential algebraic equation was first studied by Marszalek. He also studied the analysis of the partial differential algebraic equations [1]. Lucht et al. [24] studied the numerical solution and indexes of the linear partial differential equations with constant coefficients. A study about characteristics analysis and differential index of the partial differential algebraic equations was given by Martinson and Barton [5, 6]. Debrabant and Strehmel investigated the convergence of Runge-Kutta method for linear partial differential algebraic equations [7].

There are numerous LPDAEs applications in scientific areas given, for instance, in the field of Navier-Stokes equations, in chemical engineering, in magnetohydrodynamics, and in the theory of elastic multibody systems $[4,8-12]$.

On the other hand, the differential transform method was used by Zhou [13] to solve linear and nonlinear initial value problems in electric circuit analysis. Analysis of nonlinear circuits by using differential Taylor transform was given by Köksal and Herdem [14]. Using onedimensional differential transform, Abdel-Halim Hassan
[15] proposed a method to solve eigenvalue problems. The two-dimensional differential transform methods have been applied to the partial differential equations [1619]. The differential transform method extended to solve differential-difference equations by Arikoglu and Ozkol [20]. Jang et al. have used differential transform method to solve initial value problems [21]. The numerical solution of the differential-algebraic equation systems has been studied by using differential transform method [22, 23].

In this paper, we have considered linear partial differential equations with constant coefficients of the form

$$
\begin{array}{r}
A u_{t}(t, x)+B u_{x x}(t, x)+C u(t, x)=f(t, x), \\
(t, x) \in J \times \Omega,
\end{array}
$$

where $J=[0, \infty), \Omega=[-l, l], l>0$, and $A, B, C \in \mathbb{R}^{n \times n}$. In (1) at least one of the matrices $A, B \in \mathbb{R}^{n \times n}$ should be singular. If $A=0$ or $B=0$, then (1) becomes ordinary differential equation or differential algebraic equation, so we assume that none of the matrices $A$ or $B$ is the zero matrix. 


\section{Indexes of Partial Differential Algebraic Equation}

Let us consider (1), with initial values and boundary conditions given as follows:

$$
\begin{gathered}
u_{j}(t, \pm l)=0 \quad \text { for } t \in J, \\
u_{i}(0, x)=g(x) \quad \text { for } x \in \Omega,
\end{gathered}
$$

where $j \in \mathfrak{M}_{B C} \subseteq\{1,2, \ldots, n\}, \mathfrak{M}_{B C}$ is the set of indices of components of $u$ for which boundary conditions can be prescribed arbitrarily, and $i \in \mathfrak{M}_{I C} \subseteq\{1,2, \ldots, n\}, \mathfrak{M}_{\text {IC }}$ is the set of indices of components of $u$ for which initial conditions can be prescribed arbitrarily. The initial boundary value problem (IBVP) (1) has only one solution where a function $u$ is a solution of the problem, if it is sufficiently smooth, uniquely determined by its initial values (IVs) and boundary values (BVs), and if it solves the LPDAE point wise.

Definition of the indexes can be given using the following assumptions.

(i) Each component of the vectors $u, u_{t}$, and $f$ satisfy the following condition:

$$
|y(t, x)| \leq M e^{\alpha t}, \quad \alpha \geq 0, t \geq 0,
$$

where $M$ and $\alpha$ are independent of $t$ and $x$.

(ii) $(B, \xi A+C), \operatorname{Re}(\xi)>\alpha$, called as the matrix pencil, is regular.

(iii) $\left(A, \mu_{k} B+C\right)$ is regular for all $k$, where $\mu_{k}$ is an eigenvalue of the operator $\partial^{2} / \partial x^{2}$ together with prescribed BCs.

(iv) The vector $f(t, x)$ and the initial vector $g(x)$ are sufficiently smooth.

If we use Laplace transform, from assumption (ii), (1) can be transformed into

$$
B u_{\xi}^{\prime \prime}(x)+(\xi A+C) u_{\xi}(x)=f_{\xi}(x)+A g(x), \quad \operatorname{Re}(\xi)>\alpha,
$$

if $B$ is a singular matrix, then (4) is a DAE depending on the parameter $\xi$. To characterize $\mathfrak{M}_{B C}$, we introduce $j \in \mathfrak{M}_{B C}^{(\xi)} \subseteq$ $\{1,2, \ldots, n\}$ as the set of indices of components of $u_{\xi}$ for which boundary conditions can be prescribed arbitrarily.

In order to define a spatial index, we need the Kronecker normal form of the DAE (4). Assumption (iii) guarantees that there are nonsingular matrices $P_{L, \xi}, Q_{L, \xi} \in \mathbb{C}^{n \times n}$ such that

$$
\begin{gathered}
P_{L, \xi} B Q_{L, \xi}=\left(\begin{array}{cc}
I_{m_{1}} & 0 \\
0 & N_{L, \xi}
\end{array}\right), \\
P_{L, \xi}(\xi A+C) Q_{L, \xi}=\left(\begin{array}{cc}
R_{L, \xi} & 0 \\
0 & I_{m_{2}}
\end{array}\right),
\end{gathered}
$$

where $R_{L, \xi} \in \mathbb{C}^{m_{1} \times m_{2}}$ and $N_{L, \xi} \in \mathbb{R}^{m_{2} \times m_{2}}$ is a nilpotent Jordan chain matrix with $m_{1}+m_{2}=n$. $I_{k}$ is the unit matrix of order $k$. The Riesz index (or nilpotency) of $N_{L, \xi}$ is denoted by $\nu_{L, \xi}$ (i.e. $N_{L, \xi}^{\nu_{L, \xi}}=0, N_{L, \xi}^{\nu_{L, \xi}-1} \neq 0$ ).

Here, we will assume that there is a real number $\alpha^{*} \geq \alpha$ such that the index set $\mathfrak{M}_{B C}^{(\xi)}$ is independent of the Laplace parameter $\xi$, provided $\operatorname{Re}(\xi) \geq \alpha^{*}$.
Definition 1. Let $\alpha^{*} \in \mathbb{R}^{+}$be a number with $\alpha^{*} \geq \alpha$, such that for all $\xi \in \mathbb{C}$ with $\operatorname{Re}(\xi) \geq \alpha^{*}$

(1) the matrix pencil $(B, \xi A+C)$ is regular,

(2) $\mathfrak{M}_{B C}^{(\xi)}$ is independent of $\xi$, i.e., $\mathfrak{M}_{B C}^{(\xi)}=\mathfrak{M}_{B C}$,

(3) the nilpotency of $N_{L, \xi}$ is $\nu_{L} \geq 1$.

Then $v_{d, x}=2 v_{L}-1$ is called the "differential spatial index" of the LPDAE. If $v_{L}=0$, then the differential spatial index of LPDAE is defined to be zero.

If we use Fourier transform, (1) can be transformed into

$$
A \widehat{u}_{k}^{\prime}(t)+\left(\mu_{k} B+C\right) \widehat{u}_{k}(t)=\widehat{f}_{k}(t)+B \rho_{k}(t)
$$

with $\rho_{k}(t)=\left(\rho_{k 1}(t), \ldots, \rho_{k n}(t)\right)^{T}$ and

$$
\rho_{k i}(t)=0 \quad \text { for } i \in \mathfrak{M}_{B C},
$$

$$
\rho_{k j}(t)=\frac{1}{l}\left[\phi_{k}^{\prime}(x) u_{j}(t, x)-\phi_{k}(x) u_{x, j}(t, x)\right]_{x=-l}^{x=l}
$$

for $j \notin \mathfrak{M}_{B C}$, which results from partial integration of the $\operatorname{term} \int_{-l}^{l} u_{x x}(t, x) \phi_{k}(x) d x$.

If $A$ is a singular matrix, then (6) is a DAE depending on the parameter $\mu_{k}$ which can be solved uniquely with suitable ICs under the assumptions (iv) and (v). Analogous to the case of the Laplace transform, the above assumption (iv) implies that there exist regular matrices $P_{F, k}, Q_{F, k}$ such that

$$
\begin{gathered}
P_{F, k} A Q_{F, k}=\left(\begin{array}{cc}
I_{n_{1}} & 0 \\
0 & N_{F, k}
\end{array}\right), \\
P_{F, k}\left(\mu_{k} B+C\right) Q_{F, k}=\left(\begin{array}{cc}
R_{F, k} & 0 \\
0 & I_{n_{2}}
\end{array}\right) .
\end{gathered}
$$

With $R_{F, k} \in \mathbb{R}^{n_{1} \times n_{1}} \cdot N_{F, k} \in \mathbb{R}^{n_{2} \times n_{2}}$ is again a nilpotent Jordan chain matrix with Riesz index $v_{F, k}$, where $n_{1}+n_{2}=n$.

To characterize $\mathfrak{M}_{\text {IC }}$, we introduce $\mathfrak{M}_{\text {IC }}^{(k)} \subseteq\{1,2, \ldots, n\}$ as the set of indices of components of $\widehat{u}_{k}$ for which initial conditions can be prescribed arbitrarily. Therefore, we always assume in the context of a Fourier analysis of $u$ that $\mathfrak{M}_{I C}^{(k)}$ is independent of $k \in \mathbb{N}_{+}$, i.e., $\mathfrak{M}_{\text {IC }}^{(k)}=\mathfrak{M}_{\text {IC }}$.

Definition 2. Assume for $k=1,2, \ldots$ that

(1) the matrix pencil $\left(A, \mu_{k} B+C\right)$ is regular,

(2) $\mathfrak{M}_{\text {IC }}^{(k)}$ is independent of $k$, i.e., $\mathfrak{M}_{I C}^{(k)}=\mathfrak{M}_{\text {IC }}$,

(3) the nilpotency of $N_{F, k}$ is $\nu_{F, k}=\nu_{F}$.

Then the PDAE (1) is said to have uniform differential time index $v_{d, t}=v_{F}$.

The differential spatial and time indexes are used to decide which initial and boundary values can be taken to solve the problem. 


\section{Two-Dimensional Differential Transform Method}

The two-dimensional differential transform of function $w(x, y)$ is defined as

$$
W(k, h)=\frac{1}{k ! h !}\left[\frac{\partial^{k+h} w(x, y)}{\partial x^{k} \partial y^{h}}\right]_{\substack{x=0 \\ y=0}},
$$

where it is noted that upper case symbol $W(k, h)$ is used to denote the two-dimensional differential transform of a function represented by a corresponding lower case symbol $w(x, y)$. The differential inverse transform of $W(k, h)$ is defined as

$$
w(x, y)=\sum_{k=0}^{\infty} \sum_{h=0}^{\infty} W(k, h) x^{k} y^{h} .
$$

From (9) and (10), we obtain

$$
w(x, y)=\sum_{k=0}^{\infty} \sum_{h=0}^{\infty} \frac{x^{k} y^{h}}{k ! h !}\left[\frac{\partial^{k+h} w(x, y)}{\partial x^{k} \partial y^{h}}\right]_{\substack{x=0 \\ y=0}}
$$

The concept of two-dimensional differential transform is derived from two-dimensional Taylor series expansion, but the method doesn't evaluate the derivatives symbolically.

Theorem 3. Differential transform of the function $w(x, y)=$ $u(x, y) \pm v(x, y)$ is

$$
W(k, h)=U(k, h) \pm V(k, h),
$$

see [17].

Theorem 4. Differential transform of the function $w(x, y)=$ $\lambda u(x, y)$ is

$$
W(k, h)=\lambda U(k, h),
$$

see [17].

Theorem 5. Differential transform of the function $w(x, y)=$ $\partial u(x, y) / \partial x$ is

$$
W(k, h)=(k+1) U(k+1, h),
$$

see [17].

Theorem 6. Differential transform of the function $w(x, y)=$ $\partial u(x, y) / \partial y$ is

$$
W(k, h)=(h+1) U(k, h+1),
$$

see [17].

Theorem 7. Differential transform of the function $w(x, y)=$ $\partial^{r+s} u(x, y) / \partial x^{r} \partial y^{s}$ is

$$
\begin{aligned}
W(k, h)= & (k+1)(k+2) \cdots(k+r)(h+1) \\
& \times(h+2) \cdots(h+s) U(k+r, h+s),
\end{aligned}
$$

see [17].
Theorem 8. Differential transform of the function $w(x, y)=$ $u(x, y) \cdot v(x, y)$ is

$$
W(k, h)=\sum_{r=0}^{k} \sum_{s=0}^{h} U(r, h-s) V(k-r, s),
$$

see [17].

Theorem 9. Differential transform of the function $w(x, y)=$ $x^{m} y^{n}$ is

$$
W(k, h)=\delta(k-m, h-n)=\delta(k-m) \delta(h-n),
$$

see [17], where

$$
\begin{aligned}
& \delta(k-m)= \begin{cases}1, & k=m \\
0, & k \neq m,\end{cases} \\
& \delta(h-n)= \begin{cases}1, & h=n \\
0, & h \neq n .\end{cases}
\end{aligned}
$$

Theorem 10. Differential transform of the function $w(x, y)=$ $g(x+a, y)$ is

$$
W(k, h)=\sum_{p=k}^{N}\left(\begin{array}{l}
p \\
k
\end{array}\right) a^{p-k} G(p, h) .
$$

Proof. From Definition 1, we can write

$$
\begin{aligned}
& w(x, y)=\sum_{h=0}^{\infty} \sum_{k=0}^{\infty} G(k, h)(x+a)^{k} y^{h} \\
& =G(0,0)+G(0,1) y+a G(1,0)+G(1,0) x \\
& +G(0,2) y^{2}+G(1,1) x y+a G(1,1) y \\
& +a^{2} G(2,0)+2 a G(2,0) x+G(2,0) x^{2} \\
& +G(0,3) y^{3}+G(1,2) x y^{2}+a G(1,2) y^{2} \\
& +G(2,1) x^{2} y+2 a G(2,1) x y+a^{2} G(2,1) y \\
& +a^{3} G(3,0)+3 a^{2} G(3,0) x+3 a G(3,0) x^{2} \\
& +G(3,0) x^{3}+\cdots, \\
& w(x, y)=[G(0,0)+a G(1,0) \\
& \left.+a^{2} G(2,0)+a^{3} G(3,0)+\cdots\right] \\
& +x\left[G(1,0)+2 a G(2,0)+3 a^{2} G(3,0)+\cdots\right] \\
& +y\left[G(0,1)+a G(1,1)+a^{2} G(2,1)+\cdots\right] \\
& +x^{2}[G(2,0)+3 a G(3,0)+\cdots] \\
& +x y[G(1,1)+2 a G(2,1)+\cdots] \\
& +y^{2}[G(0,2)+a G(1,2)+\cdots]+\cdots
\end{aligned}
$$




$$
\begin{aligned}
= & \sum_{p=0}^{\infty} a^{p} G(p, 0)+x \sum_{p=1}^{\infty} p a^{p-1} G(p, 0) \\
& +y \sum_{p=0}^{\infty} a^{p} G(p, 1)+x^{2} \sum_{p=2}^{\infty} \frac{p !}{(p-2) ! 2 !} \\
& \times a^{p-2} G(p, 0)+x y \sum_{p=1}^{\infty} p a^{p-1} G(p, 1)+\cdots,
\end{aligned}
$$

where

$$
w(x, y)=\sum_{h=0}^{\infty} \sum_{k=0}^{\infty} \sum_{p=k}^{\infty}\left(\begin{array}{l}
p \\
k
\end{array}\right) a^{p-k} G(p, h) x^{k} y^{h}
$$

hence,

$$
W(k, h)=\sum_{p=k}^{N}\left(\begin{array}{l}
p \\
k
\end{array}\right) a^{p-k} G(p, h) .
$$

Theorem 11. Differential transform of the function $w(x, y)=$ $g(x+a, y+b)$ is

$$
W(k, h)=\sum_{p=k}^{N} \sum_{q=h}^{N}\left(\begin{array}{l}
q \\
h
\end{array}\right)\left(\begin{array}{l}
p \\
k
\end{array}\right) a^{p-k} b^{q-h} G(p, q) .
$$

Proof. From Definition 2, we can write

$$
\begin{aligned}
w(x, y)= & \sum_{h=0}^{\infty} \sum_{k=0}^{\infty} G(k, h)(x+a)^{k}(y+b)^{h} \\
= & G(0,0)+G(1,0) x+a G(1,0) \\
& +G(0,1) y+b G(0,1)+G(2,0) x^{2} \\
& +2 a G(2,0) x+a^{2} G(2,0)+G(1,1) x y \\
& +b G(1,1) x+a G(1,1) y+a b G(1,1) \\
& +G(0,2) y^{2}+2 b G(0,2) y+b^{2} G(0,2) \\
& +G(3,0) x^{3}+3 a G(3,0) x^{2}+3 a^{2} G(3,0) x \\
& +a^{3} G(3,0)+G(2,1) x^{2} y+\cdots \\
w(x, y)= & {[G(0,0)+a G(1,0)+b G(0,1)} \\
& \left.+a^{2} G(2,0)+a b G(1,1)+\cdots\right] \\
& +[G(1,0)+2 a G(2,0)+b G(1,1) \\
& \left.+3 a^{2} G(3,0)+2 a b G(2,1)+\cdots\right] x \\
& +[G(0,1)+a G(1,1)+2 b G(0,2) \\
& \left.+a^{2} G(2,1)+\cdots\right] y+\cdots
\end{aligned}
$$

$$
\begin{aligned}
= & \sum_{p=0}^{N} \sum_{q=0}^{N} a^{p} b^{q} G(p, q)+x \sum_{p=1}^{N} \sum_{q=0}^{N} p a^{p-1} b^{q} G(p, q) \\
& +y \sum_{p=0}^{N} \sum_{q=1}^{N} q a^{p} b^{q-1} G(p, q) \\
& +x^{2} \sum_{p=2}^{N} \sum_{q=0}^{N} \frac{p !}{(p-2) ! 2 !} a^{p-2} b^{q} G(p, q) \\
& +x y \sum_{p=1}^{N} \sum_{q=1}^{N} p q a^{p-1} b^{q-1} G(p, q)+\cdots .
\end{aligned}
$$

Hence, we can write

$$
\begin{aligned}
w(x, y)=\sum_{h=0}^{\infty} \sum_{k=0}^{\infty} \sum_{p=k}^{N} \sum_{q=h}^{N}\left(\begin{array}{l}
q \\
h
\end{array}\right)\left(\begin{array}{l}
p \\
k
\end{array}\right) & \\
& \times a^{p-k} b^{q-h} G(p, q) x^{k} y^{h} .
\end{aligned}
$$

Using Definition 2, we obtain

$$
W(k, h)=\sum_{p=k}^{N} \sum_{q=h}^{N}\left(\begin{array}{l}
q \\
h
\end{array}\right)\left(\begin{array}{l}
p \\
k
\end{array}\right) a^{p-k} b^{q-h} G(p, q) .
$$

Theorem 12. Differential transform of the function $w(x, y)=$ $\partial^{r+s} g(x+a, y+b) / \partial x^{r} \partial y^{s}$ is

$$
\begin{aligned}
& W(k, h)= \frac{(k+r) !}{k !} \frac{(h+s) !}{h !} \\
& \times \sum_{p=k+r}^{N} \sum_{q=h+s}^{N}\left(\begin{array}{c}
q \\
h+s
\end{array}\right)\left(\begin{array}{c}
p \\
k+r
\end{array}\right) \\
& \quad \times a^{p-k-r} b^{q-h-s} G(p, q) .
\end{aligned}
$$

Proof. Let $C(k, h)$ be differential transform of the function $g(x+a, y+b)$. From Theorem 7 , we can write that differential transform of the function $w(x, y)$ is

$$
W(k, h)=\frac{(k+r) !}{k !} \frac{(h+s) !}{h !} C(k+r, h+s),
$$

from Theorem 4, we can write

$$
\begin{array}{r}
C(k+r, h+s)=\sum_{p=k+r}^{N} \sum_{q=h+s}^{N}\left(\begin{array}{c}
q \\
h+s
\end{array}\right)\left(\begin{array}{c}
p \\
k+r
\end{array}\right) \\
\times a^{p-k-r} b^{q-h-s} G(p, q) .
\end{array}
$$


If we substitute (30) into (29), we find

$$
\begin{aligned}
W(k, h)= & \frac{(k+r) !}{k !} \frac{(h+s) !}{h !} \\
& \times \sum_{p=k+r}^{N} \sum_{q=h+s}^{N}\left(\begin{array}{c}
q \\
h+s
\end{array}\right)\left(\begin{array}{c}
p \\
k+r
\end{array}\right) \\
& \times a^{p-k-r} b^{q-h-s} G(p, q) .
\end{aligned}
$$

\section{Application}

We have considered the following PDAE as a test problem:

$$
\begin{array}{r}
\left(\begin{array}{ll}
1 & 1 \\
0 & 0
\end{array}\right) u_{t}+\left(\begin{array}{cc}
-1 & 0 \\
-1 & 0
\end{array}\right) u_{x x}+\left(\begin{array}{cc}
1 & 1 \\
-1 & 0
\end{array}\right) u=f \\
t \in[0, \infty), \quad x \in[-1,1],
\end{array}
$$

with initial values and boundary values

$$
\begin{array}{cl}
u_{1}(0, x)=x^{3}-x, & u_{2}(0, x)=x^{4}-1, \\
u_{1}(t, 1)=u_{1}(t,-1)=0, & u_{2}(t, 1)=u_{2}(t,-1)=0 .
\end{array}
$$

The right hand side function $f$ is

$$
f=\left(\left(x^{4}-1\right)(\cos t-\sin t)-6 x e^{-t},-e^{-t}\left(x^{3}+5 x\right)\right)^{T},
$$

and the exact solutions are

$$
u_{1}(t, x)=\left(x^{3}-x\right) e^{-t}, \quad u_{2}(t, x)=\left(x^{4}-1\right) \cos t .
$$

If nonsingular matrices $P_{F, k}, Q_{F, k}, P_{L, \xi}$, and $Q_{L, \xi}$ are chosen such as

$$
\begin{aligned}
& P_{F, k}=\left(\begin{array}{cc}
1 & \frac{-\mu_{k}}{\mu_{k}-1} \\
0 & \frac{1}{\mu_{k}-1}
\end{array}\right), \quad Q_{F, k}=\left(\begin{array}{ll}
0 & -1 \\
1 & -1
\end{array}\right), \\
& P_{L, \xi}=\left(\begin{array}{cc}
0 & \frac{1}{\xi+1} \\
\frac{1}{\xi+1} & -\frac{1}{\xi+1}
\end{array}\right), \quad Q_{L, \xi}=\left(\begin{array}{cc}
-\xi-1 & 0 \\
\xi & 1
\end{array}\right),
\end{aligned}
$$

matrices $P_{F, k} A Q_{F, k}$ and $P_{L, \xi} B Q_{L, \xi}$ are found as

$$
P_{F, k} A Q_{F, k}=\left(\begin{array}{cc}
1 & 0 \\
0 & 0
\end{array}\right), \quad P_{L, \xi} B Q_{L, \xi}=\left(\begin{array}{ll}
1 & 0 \\
0 & 0
\end{array}\right) .
$$

From (38), we have $N_{L, \xi}=0$ and $N_{F, k}=0$. Then the PDAE (32) has differential spatial index 1 and differential time index 1. So, it is enough to take $\mathfrak{M}_{B C}^{(\xi)}=\{1\}$ and $\mathfrak{M}_{I C}^{(k)}=\{2\}$ to solve the problem.

Taking differential transformation of (32), we obtain

$$
\begin{gathered}
(k+1) U_{1}(k+1, h)+(k+1) U_{2}(k+1, h) \\
-(h+1)(h+2) U_{1}(k, h+2)+U_{1}(k, h) \\
+U_{2}(k, h)=F_{1}(k, h), \\
-(h+1)(h+2) U_{1}(k, h+2)-U_{1}(k, h)=F_{2}(k, h) .
\end{gathered}
$$

TABLE 1: The numerical and exact solution of the test problem(32), where $u_{1}(t, x)$ is the exact solution and $u_{1}^{*}(t, x)$ is the numerical solution, for $x=0.1$

\begin{tabular}{lccc}
\hline$t$ & $u_{1}(t, x)$ & $u_{1}^{*}(t, x)$ & $\left|u_{1}(t, x)-u_{1}^{*}(t, x)\right|$ \\
\hline 0.1 & -0.0895789043 & -0.0895789043 & 0 \\
0.2 & -0.0810543445 & -0.0810543422 & 0.0000000023 \\
0.3 & -0.0733410038 & -0.0733409887 & 0.0000000151 \\
0.4 & -0.0663616845 & -0.0663616355 & 0.0000000490 \\
0.5 & -0.0600465353 & -0.0600464409 & 0.0000000944 \\
0.6 & -0.0543323519 & -0.0543322800 & 0.0000000719 \\
0.7 & -0.0491619450 & -0.0491621943 & 0.0000002493 \\
0.8 & -0.0444835674 & -0.0444849422 & 0.0000013748 \\
0.9 & -0.0402503963 & -0.0402546487 & 0.0000042524 \\
1.0 & -0.0364200646 & -0.0364305555 & 0.0000104909 \\
\hline
\end{tabular}

TABLE 2: The numerical and exact solution of the test problem(32), where $u_{2}(t, x)$ is exact solution and $u_{2}^{*}(t, x)$ is numerical solution, for $x=0.1$

\begin{tabular}{lccc}
\hline$t$ & $u_{2}(t, x)$ & $u_{2}^{*}(t, x)$ & $\left|u_{2}(t, x)-u_{2}^{*}(t, x)\right|$ \\
\hline 0.1 & -0.9949046649 & -0.9949046653 & 0.0000000004 \\
0.2 & -0.9799685711 & -0.9799685778 & 0.0000000067 \\
0.3 & -0.9552409555 & -0.9552409875 & 0.0000000320 \\
0.4 & -0.9209688879 & -0.9209689778 & 0.0000000899 \\
0.5 & -0.8774948036 & -0.8774949653 & 0.0000001637 \\
0.6 & -0.8252530813 & -0.8252532000 & 0.0000001187 \\
0.7 & -0.7647657031 & -0.7647652653 & 0.0000004378 \\
0.8 & -0.6966370386 & -0.6966345778 & 0.0000024608 \\
0.9 & -0.6215478073 & -0.6215398875 & 0.0000079198 \\
1.0 & -0.5402482757 & -0.5402277778 & 0.0000204979 \\
\hline
\end{tabular}

The Taylor series of functions $f_{1}$ and $f_{2}$ about $x=0, t=0$ are

$$
\begin{aligned}
f_{1}(t, x)= & -1+t-6 x+\frac{1}{2} t^{2}+6 x t \\
& -\frac{1}{6} t^{3}-3 x t^{2}-\frac{1}{24} t^{4}+x^{4}+x t^{3} \\
& -x^{4} t+\frac{1}{120} t^{5}-\frac{1}{4} x t^{4}+\frac{1}{720} t^{6} \\
& -\frac{1}{2} x^{4} t^{2}+\frac{1}{20} x t^{5}+\frac{1}{6} x^{4} t^{3} \\
& -\frac{1}{5040} t^{7}-\frac{1}{120} x t^{6}+\cdots \\
f_{2}(t, x)= & -5 x+5 x t-\frac{5}{2} x t^{2}-x^{3} \\
& +\frac{5}{6} x t^{3}+x^{3} t-\frac{1}{2} x^{3} t^{2}-\frac{5}{24} x t^{4} \\
& +\frac{1}{6} x^{3} t^{3}+\frac{1}{24} x t^{5}-\frac{1}{144} x t^{6} \\
& -\frac{1}{24} x^{3} t^{4}+\cdots
\end{aligned}
$$




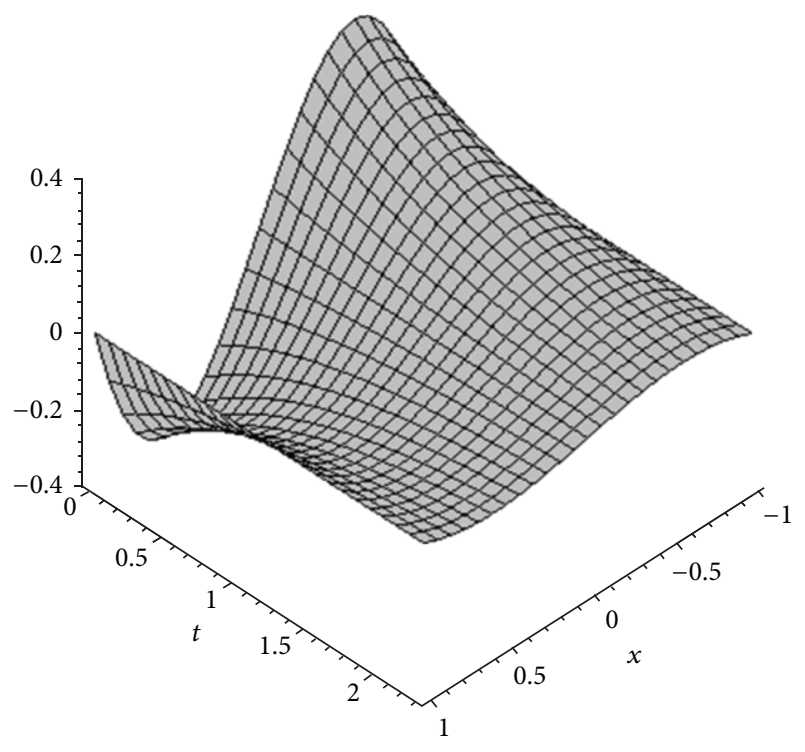

FIgURE 1: The graphic of the function $u_{1}(t, x)$ in the test problem (32).

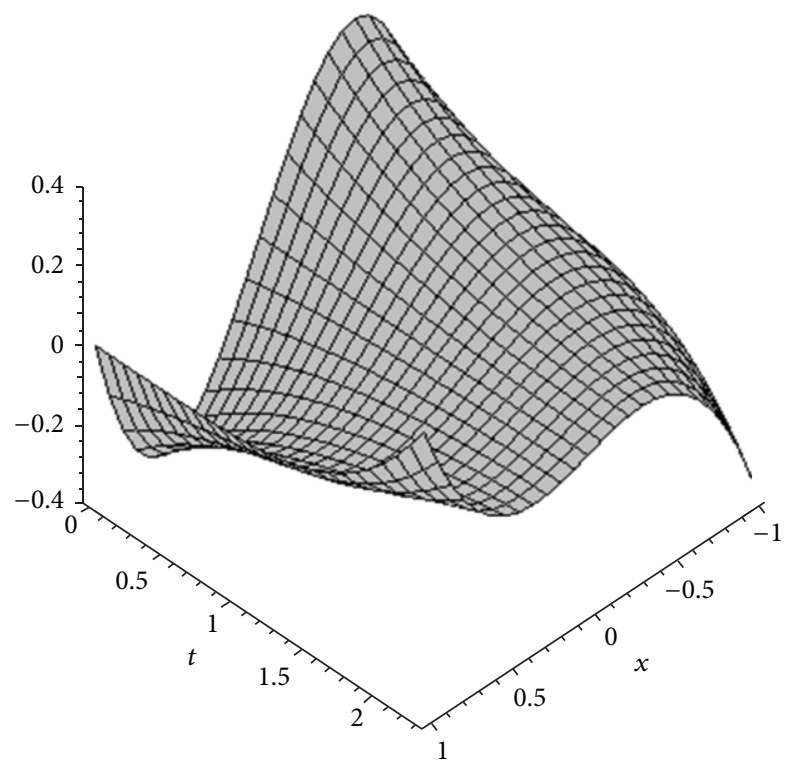

FIGURE 2: The graphic of the function $u_{1}^{*}(t, x)$ in the test problem (32).

The values $F_{1}(k, h)$ and $F_{2}(k, h)$ in (39) and (40) are coefficients of polynomials (41) and (42). If we use Theorem 3 for boundary values, we obtain

$$
\begin{gathered}
\sum_{i=0}^{7} U_{1}(j, i)=0, \quad j=0,1, \ldots, 7, \\
\sum_{i=0}^{7}(-1)^{i} U_{1}(j, i)=0, \quad j=0,1, \ldots, 7 .
\end{gathered}
$$

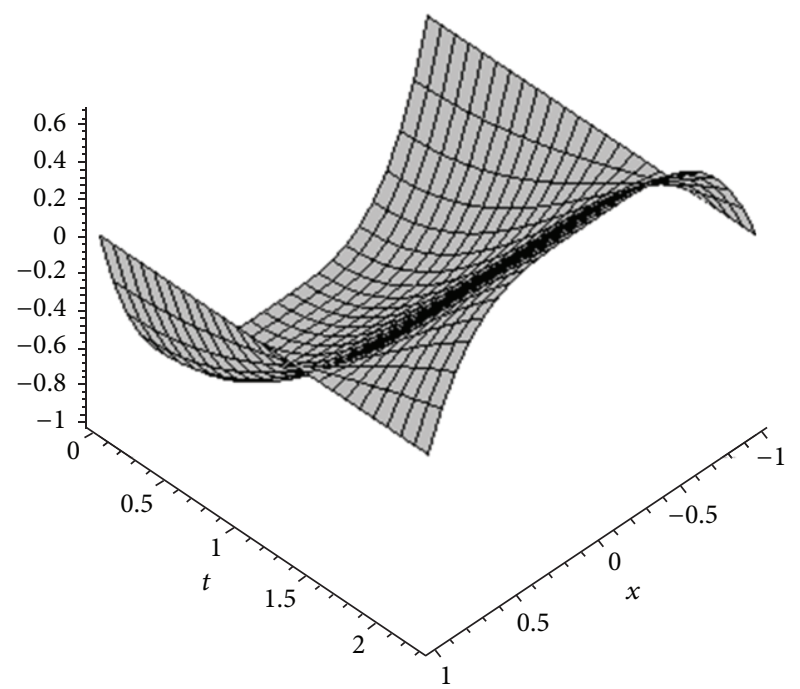

FIGURE 3: The graphic of the function $u_{2}(t, x)$ in the test problem (32).

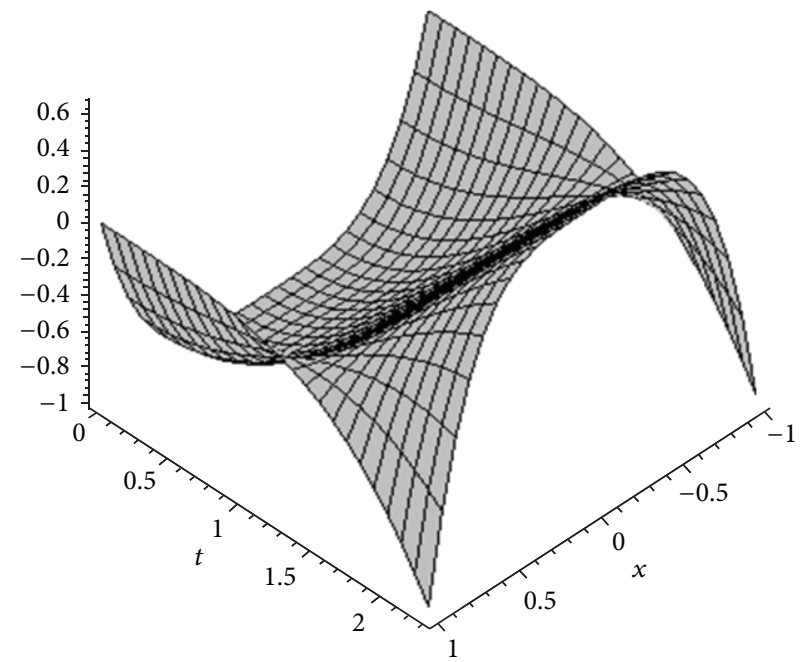

FIgURE 4: The graphic of the function $u_{2}^{*}(t, x)$ in the test problem (32).

In order to write $k=0$ and $h=0,1,2,3,4,5$ in (40), we have

$$
\begin{array}{ll}
2 U_{1}(0,2)+U_{1}(0,0)=0, & 20 U_{1}(0,5)+U_{1}(0,3)=1, \\
6 U_{1}(0,3)+U_{1}(0,1)=5, & 30 U_{1}(0,6)+U_{1}(0,4)=0, \\
12 U_{1}(0,4)+U_{1}(0,2)=0, & 42 U_{1}(0,7)+U_{1}(0,5)=0 .
\end{array}
$$

If we take $j=0$ in (43) and (44), we obtain

$$
\begin{aligned}
& U_{1}(0,0)+U_{1}(0,1)+U_{1}(0,2)+U_{1}(0,3) \\
& \quad+U_{1}(0,4)+U_{1}(0,5)+U_{1}(0,6)+U_{1}(0,7)=0 \\
& U_{1}(0,0)-U_{1}(0,1)+U_{1}(0,2)-U_{1}(0,3) \\
& \quad+U_{1}(0,4)-U_{1}(0,5)+U_{1}(0,6)-U_{1}(0,7)=0 .
\end{aligned}
$$


From (45) and (46), we find

$$
\begin{gathered}
U_{1}(0,0)=0, \quad U_{1}(0,1)=-1, \quad U_{1}(0,2)=0, \\
U_{1}(0,3)=1, \quad U_{1}(0,4)=0, \quad U_{1}(0,5)=0, \\
U_{1}(0,6)=0, \quad U_{1}(0,7)=0 .
\end{gathered}
$$

In this manner, from (40), (44), and (45), the coefficients of the $u_{1}$ are obtained as follows:

$$
\begin{array}{ccc}
U_{1}(1,0)=0, & U_{1}(1,1)=1, & U_{1}(1,2)=0, \\
U_{1}(1,3)=-1, & U_{1}(1,4)=0, & U_{1}(1,5)=0, \\
U_{1}(1,6)=0, & U_{1}(2,0)=0, & U_{1}(2,1)=-\frac{1}{2}, \\
U_{1}(2,2)=0, & U_{1}(2,3)=\frac{1}{2}, & U_{1}(2,4)=0, \\
U_{1}(2,5)=0, & U_{1}(3,0)=0, & U_{1}(3,1)=\frac{1}{6}, \\
U_{1}(3,2)=0, & U_{1}(3,3)=-\frac{1}{6}, & U_{1}(3,4)=0, \\
U_{1}(4,0)=0, & U_{1}(4,1)=-\frac{1}{24}, & U_{1}(4,2)=0, \\
U_{1}(4,3)=\frac{1}{24}, & U_{1}(5,0)=0, & U_{1}(5,1)=\frac{1}{120}, \\
U_{1}(5,2)=0, & U_{1}(6,0)=0, & U_{1}(6,1)=-\frac{1}{720},
\end{array}
$$

Using the initial values for the second component, we obtain the following coefficients:

$$
\begin{gathered}
U_{2}(0,0)=-1, \quad U_{2}(0,1)=0, \quad U_{2}(0,2)=0, \\
U_{2}(0,3)=0, \quad U_{2}(0,4)=1, \quad U_{2}(0,5)=0, \\
U_{2}(0,6)=0, \quad U_{2}(0,7)=0 .
\end{gathered}
$$

The coefficients of the $u_{2}$ can be found using (47), (48), (49), and taking $k=0,1,2, \ldots$ and $h=0,1,2, \ldots$ in (39) as follows:

$$
\begin{array}{ccc}
U_{2}(1,2)=0, & U_{2}(1,3)=0, & U_{2}(1,4)=0, \\
U_{2}(1,5)=0, & U_{2}(1,6)=0, & U_{2}(2,1)=0, \\
U_{2}(2,2)=0, & U_{2}(2,3)=0, & U_{2}(2,4)=-\frac{1}{2}, \\
U_{2}(2,5)=0, & U_{2}(3,0)=0, & U_{2}(3,1)=0, \\
U_{2}(3,2)=0, & U_{2}(3,3)=0, & U_{2}(3,4)=0, \\
U_{2}(4,1)=0, & U_{2}(4,2)=0, & U_{2}(4,3)=0,
\end{array}
$$

$$
\begin{array}{ccc}
U_{2}(4,0)=-\frac{1}{24}, & U_{2}(5,0)=0, & U_{2}(5,1)=0, \\
U_{2}(5,2)=0, & U_{2}(6,0)=\frac{1}{720}, & U_{2}(6,1)=0, \\
U_{2}(7,0)=0 . &
\end{array}
$$

If we write the above values in (39) and (40), then we have

$$
\begin{aligned}
u_{1}^{*}(t, x)= & -x+x t-\frac{1}{2} x t^{2}+x^{3}+\frac{1}{6} x t^{3} \\
& -x^{3} t+\frac{1}{2} x^{3} t^{2}-\frac{1}{24} x t^{4}-\frac{1}{6} x^{3} t^{3} \\
& +\frac{1}{120} x t^{5}-\frac{1}{720} x t^{6}+\frac{1}{24} x^{3} t^{4}+\cdots, \\
u_{2}^{*}(t, x)= & -1+\frac{1}{2} t^{2}-\frac{1}{24} t^{4} \\
& +x^{4}+\frac{1}{720} t^{6}-\frac{1}{2} x^{4} t^{2}+\cdots
\end{aligned}
$$

Numerical and exact solution of the given problem has been compared in Tables 1 and 2, and simulations of solutions have been depicted in Figures 1, 2, 3, and 4, respectively.

\section{Conclusion}

The computations associated with the example discussed above were performed by using Computer Algebra Techniques [24]. We show the results in Tables 1 and 2 for the solution of (32) by numerical method. The numerical values on Tables 1 and 2 obtained above are in full agreement with the exact solutions of (32). This study has shown that the differential transform method often shows superior performance over series approximants, providing a promising tool for using in applied fields.

\section{References}

[1] W. Marszalek, Analysis of partial differential algebraic equations [Ph.D. thesis], North Carolina State University, Raleigh, NC, USA, 1997.

[2] W. Lucht, K. Strehmel, and C. Eichler-Liebenow, "Linear partial differential algebraic equations, Part I: indexes, consistent boundary/initial conditions," Report 17, Fachbereich Mathematik und Informatik, Martin-Luther-Universitat Halle, 1997.

[3] W. Lucht, K. Strehmel, and C. Eichler-Liebenow, "Linear partial differential algebraic equations, Part II: numerical solution," Report 18, Fachbereich Mathematik und Informatik, MartinLuther-Universitat Halle, 1997.

[4] W. Lucht, K. Strehmel, and C. Eichler-Liebenow, "Indexes and special discretization methods for linear partial differential algebraic equations," BIT Numerical Mathematics, vol. 39, no. 3, pp. 484-512, 1999.

[5] W. S. Martinson and P. I. Barton, "A differentiation index for partial differential-algebraic equations," SIAM Journal on Scientific Computing, vol. 21, no. 6, pp. 2295-2315, 2000. 
[6] W. S. Martinson and P. I. Barton, "Index and characteristic analysis of linear PDAE systems," SIAM Journal on Scientific Computing, vol. 24, no. 3, pp. 905-923, 2002.

[7] K. Debrabant and K. Strehmel, "Convergence of Runge-Kutta methods applied to linear partial differential-algebraic equations," Applied Numerical Mathematics, vol. 53, no. 2-4, pp. 213229, 2005.

[8] N. Guzel and M. Bayram, "On the numerical solution of stiff systems," Applied Mathematics and Computation, vol. 170, no. 1, pp. 230-236, 2005.

[9] E. Çelik and M. Bayram, "The numerical solution of physical problems modeled as a system of differential-algebraic equations (DAEs)," Journal of the Franklin Institute, vol. 342, no. 1, pp. 1-6, 2005.

[10] M. Kurulay and M. Bayram, "Approximate analytical solution for the fractional modified $\mathrm{KdV}$ by differential transform method," Communications in Nonlinear Science and Numerical Simulation, vol. 15, no. 7, pp. 1777-1782, 2010.

[11] M. Bayram, "Automatic analysis of the control of metabolic networks," Computers in Biology and Medicine, vol. 26, no. 5, pp. 401-408, 1996.

[12] N. Guzel and M. Bayram, "Numerical solution of differentialalgebraic equations with index-2," Applied Mathematics and Computation, vol. 174, no. 2, pp. 1279-1289, 2006.

[13] J. K. . Zhou, Differential Transformation and Its Application for Electrical CircuIts, Huazhong University Press, Wuhan, China, 1986.

[14] M. Köksal and S. Herdem, "Analysis of nonlinear circuits by using differential Taylor transform," Computers and Electrical Engineering, vol. 28, no. 6, pp. 513-525, 2002.

[15] I. H. Abdel-Halim Hassan, "On solving some eigenvalue problems by using a differential transformation," Applied Mathematics and Computation, vol. 127, no. 1, pp. 1-22, 2002.

[16] F. Ayaz, "On the two-dimensional differential transform method," Applied Mathematics and Computation, vol. 143, no. 2-3, pp. 361-374, 2003.

[17] C. K. Chen and S. H. Ho, "Solving partial differential equations by two-dimensional differential transform method," Applied Mathematics and Computation, vol. 106, no. 2-3, pp. 171-179, 1999.

[18] M.-J. Jang, C.-L. Chen, and Y.-C. Liu, “Two-dimensional differential transform for partial differential equations," Applied Mathematics and Computation, vol. 121, no. 2-3, pp. 261-270, 2001.

[19] X. Yang, Y. Liu, and S. Bai, "A numerical solution of secondorder linear partial differential equations by differential transform," Applied Mathematics and Computation, vol. 173, no. 2, pp. 792-802, 2006.

[20] A. Arikoglu and I. Ozkol, "Solution of differential-difference equations by using differential transform method," Applied Mathematics and Computation, vol. 181, no. 1, pp. 153-162, 2006.

[21] M.-J. Jang, C.-L. Chen, and Y.-C. Liy, "On solving the initialvalue problems using the differential transformation method," Applied Mathematics and Computation, vol. 115, no. 2-3, pp. 145$160,2000$.

[22] F. Ayaz, "Applications of differential transform method to differential-algebraic equations," Applied Mathematics and Computation, vol. 152, no. 3, pp. 649-657, 2004.

[23] H. Liu and Y. Song, "Differential transform method applied to high index differential-algebraic equations," Applied Mathematics and Computation, vol. 184, no. 2, pp. 748-753, 2007.

[24] G. Frank, MAPLE V, CRC Press, Boca Raton, Fla, USA, 1996. 


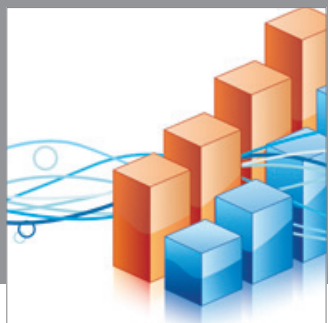

Advances in

Operations Research

mansans

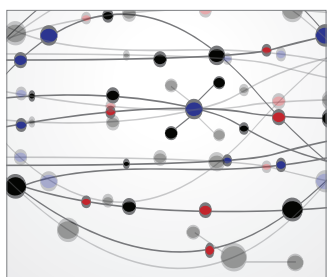

The Scientific World Journal
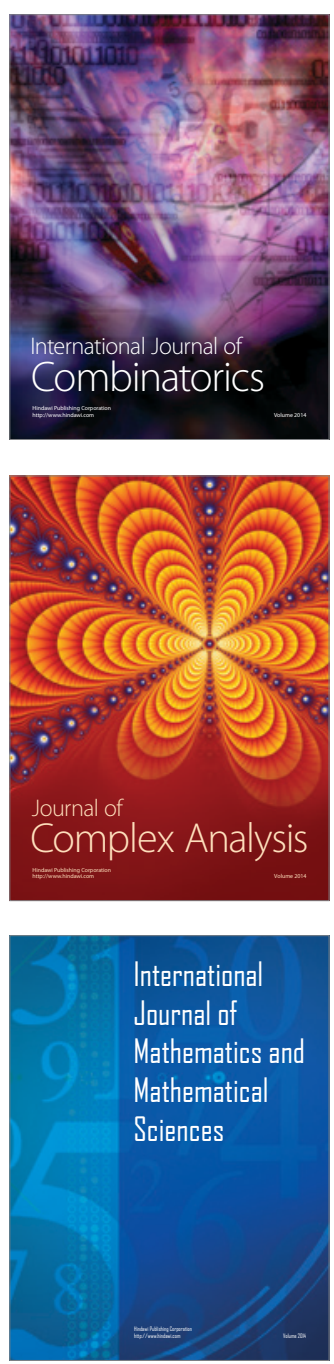
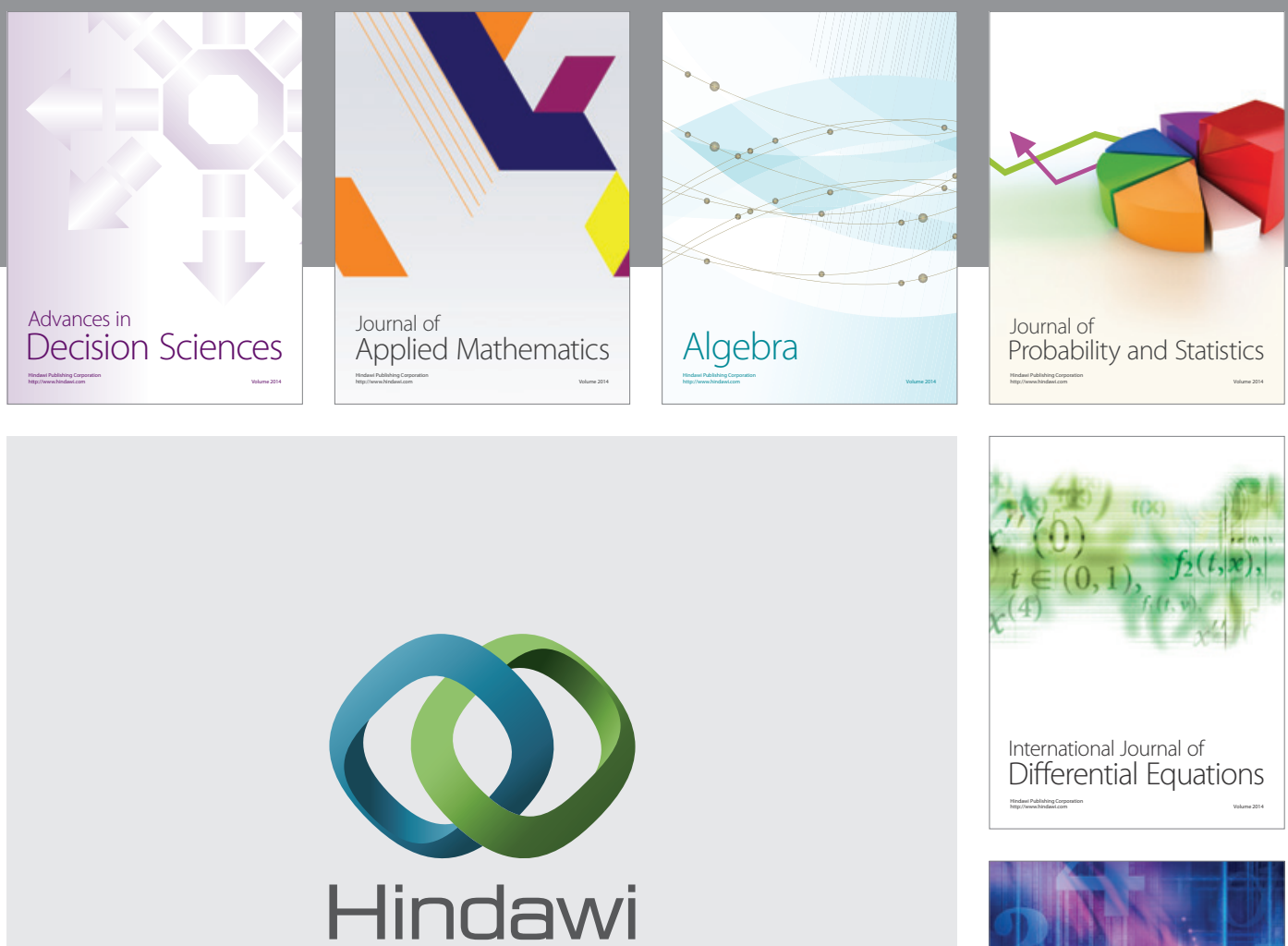

Submit your manuscripts at http://www.hindawi.com
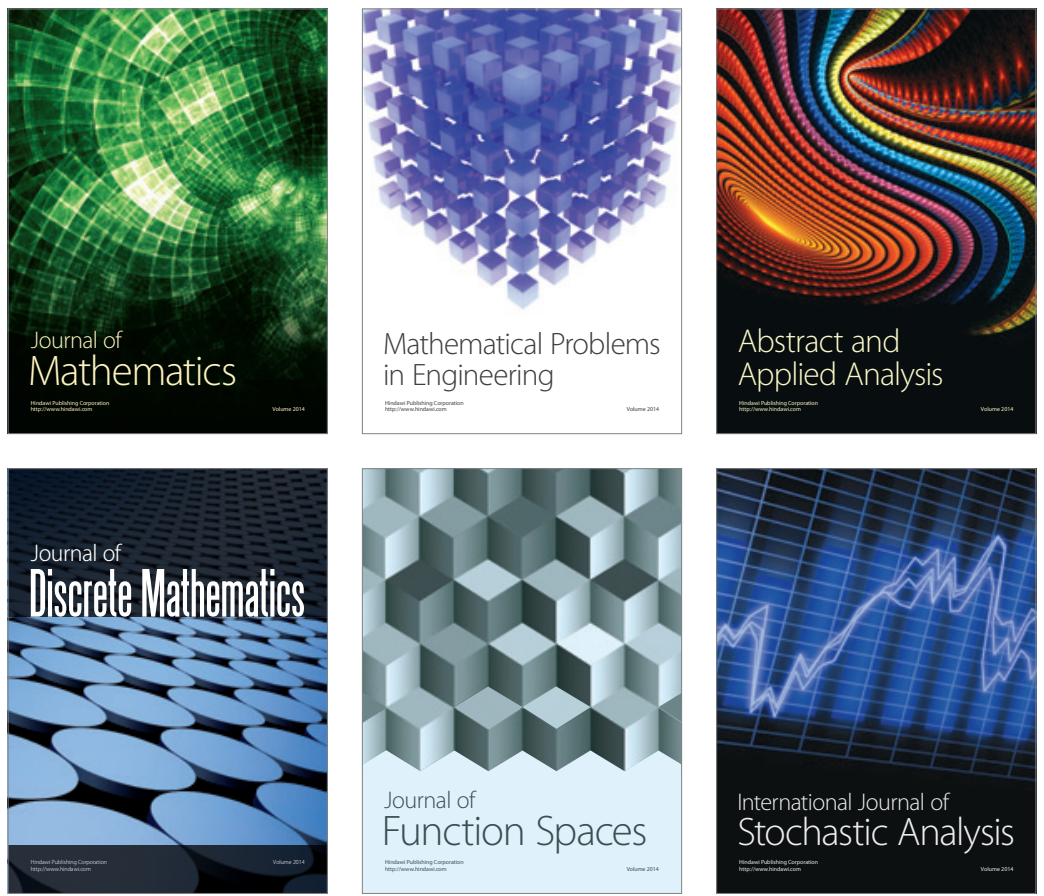

Journal of

Function Spaces

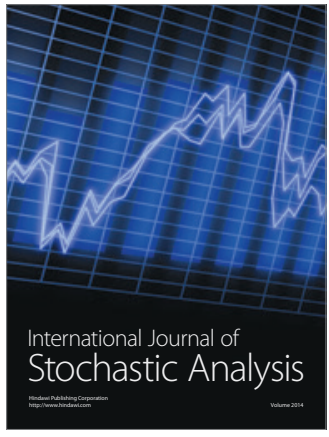

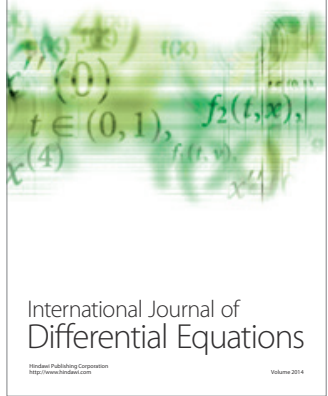
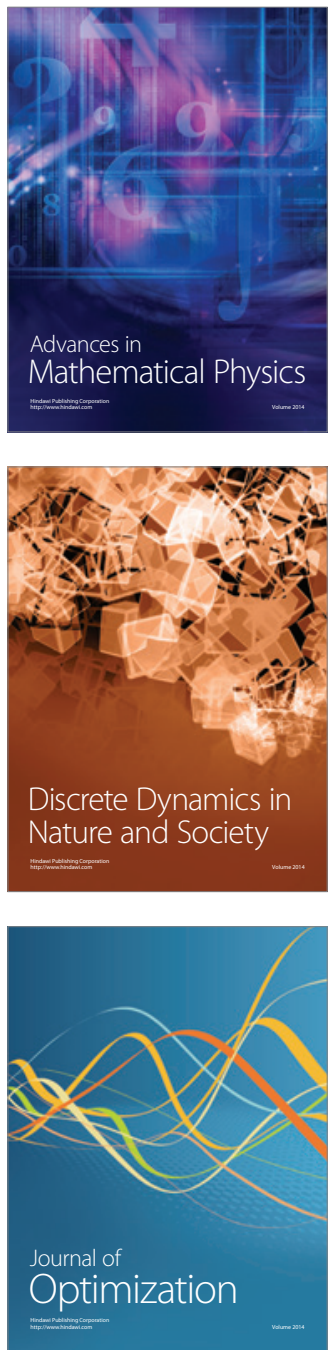